\title{
Light-controlled elimination of PD-L1+ cells
}

\author{
Judith Jing Wen Wong ${ }^{1}$, and Pål Kristian Selbo, ${ }^{1, *}$ \\ 1 Department of Radiation Biology, Institute for Cancer Research, The Norwegian Radium \\ Hospital, Oslo University Hospital, Norway; judwon@rr-research.no (J.J.W.W.); selbo@rr- \\ research.no (P.K.S.) \\ * $\quad$ Correspondence: Pål Kristian Selbo, selbo@rr-research.no; Tel.: +47-22781469
}

Received: date; Accepted: date; Published: date

Abstract: The programmed death ligand-1 (PD-L1), also known as CD274 or B7-H1, is mainly expressed on cancer cells and/or immunosuppressive cells in the tumor microenvironment (TME) and plays an essential role in tumor progression and immune escape. Immune checkpoint inhibitors (ICIs) of the PD-1/PD-L1 axis have shown impressive clinical success, however, the majority of the patients do not respond to immune checkpoint therapy (ICT). Thus, to overcome ICT resistance there is a high need for potent and novel strategies that simultaneously target both tumor cells and immunosuppressive cells in the TME. In this study, we show that the intracellular light-controlled drug delivery method photochemical internalization (PCI) induce specific and strongly enhanced cytotoxic effects of the PD-L1-targeting immunotoxin, anti-PD-L1-saporin (Anti-PDL1-SAP), in the PD-L1+ triple-negative breast cancer MDA-MB-231 cell line, while no enhanced efficacy was obtained in the PD-L1 negative control cell line MDA-MB-453. Using fluorescence microscopy, we reveal that the anti-PD-L1 antibody binds to PD-L1 on the surface of the MDA-MD-231 cells and overnight accumulates in late endosomes and lysosomes where it co-localizes with the PCI photosensitizer fimaporfin (TPCS 2 a). Moreover, light-controlled endosomal/lysosomal escape of the anti-PD-L1 antibody and fimaporfin into the cytosol was obtained. We also confirm that the breast MDA-MB-468 and the prostate PC-3 and DU-145 cancer cell lines have subpopulations with PD-L1 expression. In addition, we show that interferon-gamma strongly induce PD-L1 expression in the per se PD-L1 negative CT26.WT cells and enhance the PD-L1 expression in MC-38 cells, of which both are murine colon cancer cell lines. In conclusion, our work provides an in vitro proof-of-concept of PCIenhanced targeting and eradication of PD-L1 positive immunosuppressive cells. This lightcontrolled combinatorial strategy has a potential to advance cancer immunotherapy and should be explored in preclinical studies.

Keywords: PD-L1; saporin; photochemical internalization; immune checkpoint therapy; immunotoxin; targeted therapy; cancer; drug delivery

\section{Introduction}

Immune checkpoint therapy (ICT) represents a paradigm shift in cancer treatment as it offers durable clinical responses and improves the overall survival of patients with metastatic 


\section{Short communication}

cancer [1]. ICIs are drugs, e.g., antibodies, blocking the effect of surface inhibitory molecules, including cytotoxic T-lymphocyte-associated protein 4 (CTLA-4) and the PD-1/PD-L1 axis that control the host $\mathrm{T}$ cell activity allowing cancers to evade the host immune system [1]. Monoclonal antibodies (mAbs) blocking the PD-1 and PD-L1 interaction can restore effector $\mathrm{T}$ cell function, particularly CD8+ T cells, which have a central role in fighting cancer. PD-L1 is a transmembrane protein expressed on the surface of tumor cells and immunosuppressive stromal cells in the TME [2,3]. The PD-L1 expression on tumor cells is not exclusively responsible for the therapeutic effect of PD-1/PD-L1 blockade. Studies have indicated that PD-L1 expression on both malignant cells and immune cells contributes to and modulates $\mathrm{T}$ cell dysfunction and immune escape [4]. In addition, Kleinovink et al. demonstrated preclinically that tumors with PD-L1 negative malignant cells could be efficiently treated by PD-L1 blocking antibodies as ICT also targets PD-L1-expressing stromal cells [5]. Despite significant clinical success with PD-1/PD-L1 blockade [6-10], high rates of resistance limit their efficacy, resulting in the majority of cancer patients not responding to this type of cancer immunotherapy [11,12]. It is, therefore, a high need to develop more potent combinatorial targeting strategies that enhance cytotoxic responses in both tumor and immunosuppressive cells.

Burr et al. demonstrated that that PD-L1 is taken up into cells by endocytosis [13]. The ability of PD-L1 to undergo internalization, combined with its essential role of suppressing effector $\mathrm{T}$ cells, makes it an interesting candidate for targeted delivery of cytotoxic agents [14-16], including type I ribosome-inactivating proteins (RIP). However, a major problem for RIP I protein toxins is entrapment and enzymatic degradation in late endosomes and lysosomes $[17,18]$. Unlike the highly poisonous type II RIP ricin, saporin lacks the B-chain that facilitates cell surface binding and, in addition, an effective transport mechanism to enter the cell cytosol upon endocytosis. Therefore type I RIPs exhibit low toxicity to intact cells unless the toxin can translocate to the cytosol [28]. It has been hypothesized that RIP-based therapeutics' cytotoxic effect is caused by a small fraction of the administered dose. The majority of the administered toxin is subjected to lysosomal degradation making RIPs coupled with a targeting moiety attractive candidates to combine with PCI [28-31]. Furthermore, previous studies have indicated that $\mathrm{PCI}$ is an efficient method to enhance the delivery of immunotoxins that selectively kill cancer cells expressing CSC markers [30-33]. Interestingly, accumulating evidence suggests an association between PD-L1 expression and cancer stem cell (CSC) phenotype [19-23]. CSCs are a subset of highly aggressive therapyresistant malignant cells with stem-like features proposed to drive tumor progression, metastasis, and recurrence in patients [24,25]. Moreover, CSCs are suggested to shape the TME into an immunosuppressive landscape [19].

PCI is a modality for cytosolic release of drugs entrapped in endo/lysosomal compartments facilitating enhanced drug efficacy against intracellular targets [26,27]. The PCI technology is based on the use of the amphiphilic photosensitizer TPCS 2 /fimaporfin, which first resides at 


\section{Short communication}

the plasma membrane without fully crossing the membrane. Over time, through adsorptive endocytosis, the photosensitizer is accumulated in the membranes of endocytic vesicles that are subsequently transported to endosomes and lysosomes [28]. Upon light exposure at the appropriate wavelength and activation of the photosensitizer leads to reactive oxygen species (ROS) formation. Thus, the PCI method is based on the principles of photodynamic therapy (PDT) [29]. ROS ruptures the membrane of endosomes/lysosomes, thereby releasing the entrapped content that otherwise would have been degraded in the lysosomes [27]. The PCI technology, which was initially developed for soluble macromolecules taken up by endocytosis, has now been evaluated in combination with various therapeutics that accumulate and sequester in endosomes/lysosomes. PCI has been documented as a highly efficient drug delivery method, both in vitro and in vivo, using therapeutics such as protein toxins, immunotoxins, vaccine antigens (peptides and proteins), and chemotherapeutics such as bleomycin and doxorubicin [26,27]. The clinical relevant PCI photosensitizer, TPCS2a/fimaporfin, has been evaluated in a phase I/II combined with bleomycin (Blenoxane ${ }^{\circledR}$ ) in cancer patients with advanced and recurrent solid malignancies [30]. Here, PCI of bleomycin was found safe, tolerable, and highly efficient. Currently, TPCS 2 /fimaporfin-induced PCI of gemcitabine in inoperable bile duct cancer patients is under evaluation in a pivotal phase II trial [31]. Moreover, a phase I study evaluating PCI as a vaccination strategy for peptides (HPV) or proteins (KLH) was recently completed [32]. Hence, preclinical and clinical observations demonstrate that the PCI technology can expand the therapeutic window of drugs and reduce dose-related side effects.

Based on this, we propose that intracellular delivery using PCI of a PD-L1-targeting immunotoxin is an efficient and precision-based strategy to eradicate PD-L1 expressing tumor cells or tumor-associated immunosuppressive cells. The aim of the present study was to evaluate in vitro the potential of a PD-L1-targeting immunotoxin combined with PCI as a therapeutic strategy to target and eliminate PD-L1 expressing tumor and immunosuppressive cells. By using flow cytometry and fluorescence microscopy, we show that the triple-negative breast cancer cell line MDA-MB-231 has a high cell surface expression of PD-L1, in line with Latchman et al. [33]. Co-localization, observed as yellow fluorescing puncta, of LysoTracker Red and anti-PD-L1-AlexaFluor488 (a green fluorescing $\mathrm{mAb}$ substitute for anti-PD-L1-saporin) indicate transport of PD-L1 to late endosomes and lysosomes. Furthermore, the PD-L1-targeting $\mathrm{mAb}$ was found to co-localize with the endosome/lysosome-targeting PCI-photosensitizer fimaporfin/TPCS2a. After PCI, both antiPD-L1 mAb and fimaporfin were released into the cytosol. As an in vitro proof-of-concept study, we provide evidence that PD-L1 is transported to lysosomes and that PCI of a PD-L1targeting immunotoxin anti-PD-L1-saporin is specific and efficient in the pico-nanomolar concentrations. Our results suggests a novel light-controlled drug delivery strategy for efficient and safe elimination of PD-L1 expressing immunosuppressive cells in the TME and lays the groundwork for further preclinical explorations. 


\section{Material and methods}

\section{Cell lines and cultivations}

The human breast cancer cell lines MDA-MB-231 (ATCC HTB-26), MDA-MB-468 (ATCC HTB-132) and MDA-MB-453 (ATCC HTB-131), the human prostate cancer cell lines PC-3 (ATCC CRL-1435) and DU145 (ATCC HTB-81), the pancreatic cancer cell line PANC-1 (ATCC CRL-1469), and the murine colon cancer cell line CT26.WT (ATCC CRL-2638) were all obtained from the American Type Culture Collection (ATCC, Manassas, VA, USA). The murine colon cancer cell line MC-38 (ENH204-FP) was obtained from Kerafast (Boston, MA, USA). The MDA-MB-231, PC-3, DU145, and CT26.WT cells were maintained in RPMI-1640 culture media (R8758, Sigma-Aldrich, Saint-Louis, MO, USA), whereas MDA-MB-468, MDAMB-453, PANC-1, and MC-38 were maintained in DMEM (BE12-604F/U1, Lonza Group, Basel, Switzerland). The DMEM media for MC-38 was supplemented with $10 \mathrm{mM}$ HEPES (H0887, Sigma-Aldrich). All culture media were supplemented with $10 \%$ fetal bovine serum (ThermoFisher Scientific, Waltham, MA, USA), $100 \mathrm{U} / \mathrm{mL}$ penicillin, and $100 \mu \mathrm{g} / \mathrm{mL}$ streptomycin (Sigma-Aldrich). The cells were mycoplasma negative and maintained in a humidified incubator at $37{ }^{\circ} \mathrm{C}$ with $5 \% \mathrm{CO}_{2}$.

\section{Drugs and chemicals}

The ICI mAb atezolizumab, a PD-L1 inhibitor (Tecentriq, Roche, Basel, Switzerland), was kindly provided by the Hospital Pharmacy at Oslo University Hospital, Rikshospitalet (Oslo, Norway), and stored at $4{ }^{\circ} \mathrm{C}$. The photosensitizer TPCS 2 (fimaporfin) was kindly provided by PCI Biotech AS (Oslo, Norway) and kept in a stock solution of $0.35 \mathrm{mg} / \mathrm{mL}$ in polysorbate 80, $2.8 \%$ mannitol, $50 \mathrm{mM}$ Tris, $\mathrm{pH} 8.5$ and stored in dark at $4{ }^{\circ} \mathrm{C}$. MTT (3-(4,5-dimethylthiazol2-yl)-2,5-diphenyl tetrazolium bromide) (Sigma-Aldrich) for the MTT assay was dissolved in PBS in stock concentration $5 \mathrm{mg} / \mathrm{mL}$ and stored at $4{ }^{\circ} \mathrm{C}$ in the dark. All work involving $\mathrm{TPCS}_{2 \mathrm{a}}$ was performed under subdued light.

\section{Immunotoxin}

The immunotoxin anti-human PD-L1-saporin (Anti-PD-L1-SAP, BETA-014) was generously provided by Advanced Targeting Systems (ATS, Carlsbad, CA, USA). The targeted toxin is a $295 \mathrm{kDa}$ conjugate between a rabbit polyclonal antibody to human PD-L1 and the secondary conjugate streptavidin-saporin. The non-targeting toxin streptavidin-saporin (IT-27), from here named saporin, was also obtained from ATS.

\section{Photochemical internalization (PCI) of anti-PD-L1-saporin}

MDA-MB-231 ( $2 \times 10^{3}$ cells/well) and MDA-MB-453 ( $8 \times 10^{3}$ cells/well) were seeded in 96-well plates (Nunc) and allowed to attach overnight. The cells were then co-incubated with TPCS $2 a$ and anti-PD-L1-saporin or saporin as indicated for 18 hours. The TPCS 2 concentration was $0.2 \mu \mathrm{g} / \mathrm{mL}$ and $0.4 \mu \mathrm{g} / \mathrm{mL}$ in MDA-MB-231 and MDA-MB-453, respectively. The cells were 


\section{Short communication}

subsequently washed twice with PBS and chased 4 hours in drug-free medium to remove membrane-bound TPCS 2 before illumination. The PCI-photosensitizer fimaporfin/TPCS ${ }_{2 a}$ was activated using the LumiSource lamp (PCI Biotech AS, Oslo, Norway) emitting blue light $\left(\lambda_{\max }=435 \mathrm{~nm}\right)$ as previously described [34].

\section{Cytotoxicity assay}

Viability was evaluated 48 hours post-light exposure using the MTT-assay as previously described [34]. In brief, the cells were incubated with $0.25 \mathrm{mg} / \mathrm{mL}$ MTT in culture media for up to 4 hours. The culture media was removed, and the crystals were solubilized using DMSO before the MTT activity (absorbance) was measured at $570 \mathrm{~nm}$ [34].

\section{Flow cytometry}

Flow cytometry was performed on live cells to assess the cell surface membrane expression of PD-L1. The cells were detached by trypsin and washed with PBS once. CellTrace ${ }^{\mathrm{TM}}$ Violetstained cells (Thermo Fisher Scientific) were added to all samples prior to antibody staining to serve as an internal control. The samples were blocked with $0.5 \%$ BSA for $10 \mathrm{~min}$ on ice and incubated with the primary mAbs biotin-anti-human CD274 (B7-H1, PD-L1) (clone 29E.2A3, BioLegend, San Diego, CA, USA) or biotin anti-mouse CD274 (clone 10F.9G2, BioLegend) in $0.5 \%$ BSA for 20 min on ice. The samples were washed with PBS once and further incubated with streptavidin-AlexaFluor488 (BioLegend) in 0.5\% BSA for 20 min on ice, washed, and immediately analyzed on a flow cytometer LSRII (Becton Dickinson, Franklin Lakes, NJ, USA) as previously described [35]. Data were processed using the FlowJo software version 10.7.1 (Treestar, OR, USA).

\section{IFN- $\gamma$ treatment of murine colorectal cancer cells}

MC-38 and CT26.WT (1.5 $\times 10^{5}$ cells/well) were seeded in 6-well plates (Nunc) and allowed to attach overnight. The cells were then incubated with $100 \mathrm{U} / \mathrm{mL}$ mouse recombinant IFN- $\gamma$ (Invitrogen, Carlsbad, CA, USA) for 24 hours and subjected to flow cytometry analysis of PD-L1 expression as described above.

\section{Intracellular localization of PD-L1 and TPCS ${ }_{2 a}$ before and after light exposure (PCI)}

The binding, uptake, and intracellular localization of PD-L1 before and after PCI were evaluated by live-cell fluorescence microscopy in the MDA-MB-231 cells. Cells $\left(3 \times 10^{5}\right)$ were seeded on coverslips in 48-well plates (Nunc), allowed to attach overnight, and washed once with PBS. The cells were then incubated with biotin-anti-human CD274/PD-L1 (BioLegend) on ice for $20 \mathrm{~min}$, washed twice with PBS, and further incubated with streptavidinAlexaFluor488 (BioLegend) on ice for $20 \mathrm{~min}$. The cells were either evaluated immediately after incubation to visualize the membrane-bound PD-L1 or allowed to internalize for 18 hours. For internalization experiments, fresh culture media containing $0.4 \mu \mathrm{g} / \mathrm{mL}$ TPCS 2 was added to the samples and co-incubated with streptavidin-AlexaFluor488. At the end of the 


\section{Short communication}

incubation (18 hours), the cells were then washed twice with PBS, and chased for 4 hours in drug-free media to remove membrane-bound TPCS 2 a. Image acquisition was performed either pre- or $\sim 1$ hour post-blue light exposure (100 seconds). The cells were incubated with LysoTracker ${ }^{\mathrm{TM}}$ Red DND-99 (Thermo Fisher Scientific) for $30 \mathrm{~min}$ before image acquisition to visualize the acidic organelles. Hoechst 33342 (Thermo Fisher Scientific) was incubated for 15-20 minutes to visualize the nuclei. Image acquisition by a Zeiss Axioplan epifluorescence and phase contrast microscope using the 63x/NA1.4 PlanApo objective (Carl Zeiss AG, Oberkochen, Germany) and image processing and analysis were performed by the AxioVision Analysis software program (Carl Zeiss AG) as previously described [34].

\section{Statistical analyses}

Statistical analyses were performed using two-sided t-test SigmaPlot 14.0 (Systat Software, Inc., San Jose, CA, USA). Statistical significance was set at $\mathrm{p} \leq 0.05$.

\section{Results}

\section{Evaluation of cell membrane PD-L1 expression in breast, prostate, and pancreatic carcinoma cell lines}

Plasma membrane expression and internalization of PD-L1 is a prerequisite for PCI-based targeting of this protein. First, we therefore screened the PD-L1 surface expression in different human cancer cells; three triple-negative breast cancer cell lines (MDA-MB-231, MDA-MB-468, MDA-MB-453), two prostate cancer cell lines (DU145, PC-3), and one pancreatic cancer cell line (PANC-1) (Fig. 1A). To prevent endocytosis, live cells were incubated with the primary and secondary antibody on ice and subsequently subjected for flow cytometry analysis. Several publications indicate high PD-L1 expression in MDA-MB$231[33,36]$. Indeed, our flow cytometry data show a 10-fold increase in median fluorescence intensity (MFI) in the MDA-MB-231 cells compared to the secondary antibody alone (Fig. 1B). The minor overlap with the secondary antibody indicates that near $100 \%$ of the cells are PD-L1 positive. A 2-fold increase was detected in both of the prostate cancer cell lines (PC-3 and DU145). In the MDA-MB-453, MDA-MB-468, and PANC-1, the fluorescence intensity was comparable to the secondary antibody alone, indicating low (MDA-MB-468) or very low/no (MDA-MB-453 and PANC-1) PD-L1 membrane expression. As the MDA-MB-231 cells had the highest PD-L1 expression, this cell line was selected for further analysis to provide proof-of-concept of the PCI-based PD-L1-targeting strategy.

Anti-PD-L1 mAb binds to PD-L1 on the surface and accumulates in late endosomes and lysosomes

To perform PCI-based intracellular delivery of the anti-PD-L1-targeting immunotoxin, it is crucial that the PD-L1-targeting mAb first binds to PD-L1 and subsequently is taken up by the targeted cells, and localizes to endosomes and/or lysosomes. Thus, surface binding, 


\section{Short communication}

cellular uptake, and intracellular localization of the PD-L1-targeting mAb (anti-PD-L1AF488) and its co-localization with LysoTracker Red was first studied using fluorescence microscopy. A similar staining procedure as for flow cytometry was used, and the images were acquired immediately at the end of incubation. Live-cell fluorescence microscopy confirmed the binding of the PD-L1-targeting mAb and thereby PD-L1 expression on the surface of MDA-MB-231 cells (Fig. 2A). Image acquisition of the MDA-MB-231 cells 24 hours later revealed fluorescent puncta of anti-PD-L1-AF488 (green), which co-localized (yellow/orange) with the fluorescent marker LysoTracker Red that stains acidic organelles (endosomes and lysosomes) (Fig. 2B). Eng et al. have previously demonstrated that the photosensitizer fimaporfin/TPCS 2 co-localizes with LysoTracker Green in MDA-MB-231 cells [37]. The anti-PD-L1-AF488/PD-L1 is internalized and co-localized with LysoTracker Red in late acidic endosomes and lysosomes over time, which is optimal for performing PCIbased targeting of this immune-checkpoint protein.

\section{Light-activation and PCI-induced cy tosolic delivery of internalized anti-PD-L1 mAb}

To achieve endo/lysosomal escape using PCI, which includes photochemical-induced damage of endo/lysosomal membranes, co-localization of the PCI-photosensitizer and the immunotoxin in these vesicles is required in advance of light activation. For that reason, the co-localization of fimaporfin/TPCS 2 and anti-PD-L1-AF488 (in these experiments functioning as a substitute for the immunotoxin) in MDA-MB-231 cells before and after PCI was studied using fluorescence microscopy. Overnight incubation of fimaporfin and the PD-L1 antibody, including wash and chase as in the PCI procedure, revealed fluorescent puncta of both drugs with a high degree of intracellular co-localization (yellow/orange signal of merged images) between the anti-PD-L1-AF488 (green) and fimaporfin/TPCS $2 \mathrm{a}$ (red), indicating colocalization in late endosomes and lysosomes. (Fig. 2C). Strikingly, $\sim 1$ hour post-light exposure, the microscopy images revealed a diffuse fluorescence throughout the cytosol of both fluorochromes, indicating a PCI-induced endosomal/lysosomal escape of both the PDL1 antibody and the PCI-photosensitizer (Fig. 2C).

\section{Light-enhanced cytotoxic responses of anti-PD-L1-saporin in MDA-MB-231}

To demonstrate in vitro proof-of-concept, the PD-L1 targeting immunotoxin, anti-PD-L1saporin, was evaluated in combination with PCI in the PD-L1 high expressing MDA-MB-231 cells. Two different PCI protocols were explored; (I) an 18 hours pre-incubation with fimaporfin/TPCS 2 a prior to a 4 hours incubation of the immunotoxin during the chase period before light exposure. This protocol was compared with the second protocol (II), which included an 18 hours co-incubation of the immunotoxin with fimaporfin/TPCS 2 (Fig. 3A). Both protocols have previously been evaluated and found to be efficient at enhancing cytotoxic effects of, e.g., CD133 or EGFR-targeting toxins [38,39]. The " 4 hours incubation" protocol (I) did not result in any PCI effects, indicating that the cellular uptake of PD-L1 is relatively slow as compared to CD133 and EGFR [38,39]. Strikingly, a light-dose dependent 


\section{Short communication}

and strongly enhanced cytotoxicity by PCI of $10 \mathrm{pM}$ immunotoxin was observed with 18 hours incubation compared to PCI of the non-targeted control (saporin) (Fig. 3A). Using a 10fold higher concentration of the immunotoxin $(100 \mathrm{pM})$ did not enhance the response in the PD-L1 negative MDA-MB-453 cells compared to PCI of saporin or PDT (Fig. 3B).

Independent of PCI, the specificity and cytotoxic potential of the immunotoxin, anti-PD-L1saporin, was further evaluated and compared with the non-targeting toxin saporin. Increasing concentrations of anti-PD-L1-saporin or saporin were incubated for 18 hours in the MDA-MB-231 cells (Fig. 3C). The non-targeting toxin did not induce any substantial cytotoxicity (<10\% viability decrease) over the entire concentration range of $1-10,000 \mathrm{pM}$ in the MDA-MB-231 cells. On the other hand, the PD-L1-targeting immunotoxin induced a $44 \%$ and a $75 \%$ reduction in cell viability at $1000 \mathrm{pM}$ and $10,000 \mathrm{pM}$, respectively. To further validated the specificity and efficacy of anti-PD-L1-saporin, concentration-dependent PCI experiments in MDA-MB-231 cells using a fixed light dose (110 seconds $\left.\approx 0.77 \mathrm{~J} / \mathrm{cm}^{2}\right)$ corresponding to $\sim 50 \%$ reduction in viability with photochemical treatment alone were performed (Fig. 3D). Enhanced cytotoxicity was observed after PCI of the immunotoxin. However, compared to photochemical treatment alone, no increased cytotoxic effects was found after PCI of the non-targeting toxin (saporin) across all concentrations, indicating a selective cytotoxic efficacy can be achieved by PD-L1 targeting.

\section{Blocking PD-L1 with atezolizumab does not enhance PDT efficacy}

To evaluate if PD-L1 blockade in MDA-MB-231 cells affected viability or if combining PD-L1 blockade with PDT enhanced the cytotoxicity, we used the clinically approved anti-PD-L1 $\mathrm{mAb}$ atezolizumab (Tecentriq ${ }^{\circledR}$ ). MDA-MB-231 cells were either incubated with atezolizumab alone or co-incubated with the photosensitizer fimaporfin/TPCS 2 , subsequently washed, chased, and illuminated as in the PCI protocol. Both increasing atezolizumab concentrations combined with a fixed light dose (110 seconds light exposure $\approx$ $0.77 \mathrm{~J} / \mathrm{cm}^{2}$ ) and increasing light exposure times combined with a fixed atezolizumab concentration $(1 \mu \mathrm{M})$ were evaluated (Fig. 3E). At the highest atezolizumab concentration (5 $\mu \mathrm{M}$ ), the viability was reduced by $\sim 35 \%$, whereas lower concentrations (e.g., $100 \mathrm{nM}$ ) did not reduce the cell viability. Passariello et al. have previously reported a $\sim 25 \%$ reduction in viability in MDA-MB-231 cells after $100 \mathrm{nM}$ atezolizumab incubation for 72 hours [40]. In our experimental set-up, we used a considerably shorter (18 hours) incubation time, corresponding to the same incubation time in the PCI protocol. No enhanced fimaporfin efficacy was achieved by combining PD-L1 blockade, indicating that the PCI-enhanced toxicity of anti-PD-L1-saporin is due to the RIP activity of the toxin and not induced by binding of atezolizumab to PD-L1 .

\section{IFN- $\gamma$ induced PD-L1 expression in murine colon cancer cell lines}

Freeman et al. first demonstrated that IFN- $\gamma$ induces expression of PD-L1 [41]. The immunotoxin evaluated here is based on a polyclonal antibody recognizing the human PD- 


\section{Short communication}

L1. To further evaluate the concept in an immunocompetent model, an immunotoxin targeting the murine PD-L1 is necessary. The murine colon cancer cell lines MC-38 and CT26.WT were selected and evaluated for PD-L1 expression using flow cytometry in live cells (Fig. 4A). A 2.2-fold increase in median fluorescence intensity indicating PD-L1 expression was detected in MC-38 cells, whereas the PD-L1 expression was very low in CT26.WT cells (1.2-fold) (Fig. 4B). An incubation with IFN- $\gamma$ for 24 hours induced a 2.4fold $(\mathrm{p}<0.001)$ and $\sim 3.4$-fold $(\mathrm{p}<0.05)$ higher PD-L1 expression in in the CT26.WT and MC38 cells, respectively. These results concur with other studies $[5,41,42]$. Thus, both murine cell lines can be used as a model for a PD-L1 targeting immunotoxin in future studies.

\section{Discussion}

The majority of cancer patients do not respond to ICT, and therefore there is a high need for novel rational strategies for overcoming resistance to this type of cancer immunotherapy. It has been suggested to look beyond checkpoint blockade, which may include combinatorial therapies that both are cytotoxic and eliminate cells that drive innate and adaptive immune suppression within the TME [43]. PD-L1 is highly expressed on tumor cells and immune cells in the TME, including DCs, macrophages, myeloid-derived suppressor cells (MDSCs), and Tregs [44]. All these immunosuppressive cell populations are, therefore, potential targets for cytotoxic attack with anti-PD-L1-aiming therapeutics.

This study demonstrates the in vitro proof-of-concept of a novel cytotoxic ICT strategy for the light-controlled targeting and elimination of PD-L1 positive cells. We show that PCI of an anti-human PD-L1 immunotoxin, anti-PD-L1-saporin, is a highly efficient strategy for eliminating cancer cells overexpressing PD-L1. In line with other studies, we confirm that PD-L1 is internalized in PD-L1+ cells [13-16]. Over time, the anti-PD-L1 mAb co-localizes with the lysosomotropic PCI photosensitizer fimaporfin/TPCS2a. In addition, co-localization of the PD-L1-tageting antibody with LysoTracker suggest that a fraction of internalized PDL1 is transported to the lysosomes for degradation, and not only via recycling endosomes as previously shown [13]. After PCI, the fluorescence of TPCS $2_{a}$ and AF488-labeled PD-L1 antibody becomes diffuse, indicating endo-/lysosomal escape and cytosolic release. Our results indicate that synergistic cytotoxic effects using PCI of the anti-PD-L1-targeting immunotoxin in the PD-L1 overexpressing human triple-negative breast cancer cell line MDA-MB-231 can be achieved in picomolar to nanomolar concentrations in MDA-MB-231 cells. Our data also provide evidence of specificity of the immunotoxin as treatment with unconjugated saporin did not induce any cytotoxic responses as compared to anti-PD-L1saporin in the MDA-MB-231 cells. Moreover, PCI of anti-PD-L1-saporin was 50-fold more potent in reduction of cell viability than PCI of saporin. Lack of PCI-enhanced efficacy of anti-PD-L1-saporin was demonstrated in the PD-L1-negative control cell line MDA-MB-453, further validating the PD-L1-specificity of the immunotoxin. 


\section{Short communication}

Several groups have demonstrated the rationale of cytotoxic antibody-based targeting of immune checkpoints. One of the earliest reports by Tazzari et al. evaluated a single-chain fragment variable antibody ( $\mathrm{scFv}$ ) against CTLA-4 linked to saporin. The efficacy was demonstrated on activated $\mathrm{T}$ lymphocytes in vitro and in vivo as a strategy to prevent acute graft versus host disease (GVHD) and transplanted organ rejection [45]. Targeting of this CTLA-4-based immunotoxin was also suggested as a possible strategy for cancer treatment; however, preclinical data indicate that CTLA-4 has a significant role in regulating $\mathrm{T}$ cell responses to self-antigens [2], and systemic elimination of CTLA-4 positive cells may cause a high risk for severe adverse effects and even death. Moreover, clinical observations in cancer patients treated with anti-human CTLA- 4 mAbs have shown that immune-related adverse events (ir AEs) commonly occur $[2,46]$.

The CTLA-4 or PD-1/PD-L1 axis regulates T-cell activation; however, these immune checkpoints modulate the T-cell immune response at different levels. CTLA-4 controls T cell activation in the lymphoid organs, whereas PD-1 is a negative regulator of T-cell primarily within the peripheral tissues and in the TME through interaction with PD-L1 [1,47]. As PD-L1 is mainly expressed on cancer cells or stromal immunosuppressive cells in the TME, targeting the PD-1/PD-L1 axis provides a more restricted spectrum of adverse effects, as demonstrated in clinical studies [47], and is, therefore, suitable for PCI-targeting. Moreover, with a few exceptions, normal human tissues rarely express PD-L1 protein on the cell surface [2]. Given that the PCI method enables spatiotemporal controlled drug delivery [27], we suggest that PCI-based cytotoxic targeting of PD-L1 positive cells in the TME of unresectable tumors has the potential of being both a safe and efficient method for targeting of immune suppressive cells due to; 1 ) The combo is non-toxic in dark and dependent on in situ lightcontrolled activation; 2) The PCI-photosensitizer accumulates more in malignant than in normal tissues [28,48]; 3) The anti-PD-L1 antibody provide specific targeting and uptake of the toxin cargo in PD-L1+ cells. However, this needs to be confirmed in vivo.

Li et al. provided evidence of an anti-human PD-L1 antibody conjugated to a potent antimitotic drug in syngeneic models with tumor cells expressing human PD-L1 [14]. The antibody-drug conjugate was shown to internalize; however, targeting the glycosylated domain of PD-L1 is crucial for intracellular uptake [14]. PD-L1 antibody conjugated to the chemotherapy drug doxorubicin and doxorubicin nanoparticles have also shown promising results $[49,50]$.

Interestingly, the principle of a PD-L1 targeting immunotoxin, based on cucurmosin, has been demonstrated by Zhang et al. [51]. As saporin used in our study, cucurmosin is also a type I ribosome-inactivating protein (RIP) that potently inhibits protein synthesis but lacks an effective mechanism to enter cytosol upon endocytosis [52]. The FDA-approved anti-PDL1 mAb durvalumab, commercially known as Imfinzi® by AstraZeneca, was conjugated to cucurmosin. The immunotoxin was highly potent in the picomolar range in vitro. The immunotoxin's efficacy was further evaluated in an immunodeficient model. In the 


\section{Short communication}

immunotoxin-treated animals, an initial tumor growth delay was observed. However, poor tumor penetration due to the size was identified as an obstacle. The size of the immunotoxin by Zhang et al. was approximately $230 \mathrm{kDa}$. Of relevance, the anti-PD-L1-saporin used in this study is $295 \mathrm{kDa}$. Based on our group's previous experience, antibody-drug-conjugates of this size combined with PCI have indicated modest anti-tumor activity after PCI in vivo. The relatively large molecular size of these antibodies has been suggested to reduce the tumor penetration capacity and thereby therapeutic outcome [38,53]. One way of increasing tumor penetration capacity of immunotoxins is to replace the full-size receptor/ligand-targeting antibody with a scFv. We previously demonstrated that this strategy was, in combination with PCI, a specific and efficient anti-tumor strategy for targeting CSPG4-expressing melanoma and triple-negative breast cancer cells [37,54].

Moreover, in the context of in vivo preclinical testing, the use of anti-PD-L1-saporin in the current study is limited to human PD-L1 expressing cancer cells. Previous studies demonstrate that $\mathrm{T}$ cells are essential to achieve curative PCI effects $[55,56]$. We propose that PCI-based intracellular delivery of PD-L1 targeting immunotoxins is a feasible strategy to locally eradicate PD-L1 positive tumor cells and have the potential to eliminate immunesuppressive stromal cells expressing PD-L1 on their surface in the TME. We, therefore, aim to develop a murine version of the immunotoxin with a favorable size as this will be necessary to evaluate this strategy's full potential in immunocompetent syngeneic tumor models.

In conclusion, we demonstrate for the first time that PCI promotes strong cytotoxic effects of a PD-L1 targeting immunotoxin in PD-L1 expressing cancer cells. PCI of the immunotoxin is superior to PCI of the non-targeted toxin or the immunotoxin alone, suggesting that this light-controlled, combinatorial cytotoxic ICT have the potential of being a specific, safe and potent therapeutic strategy to target and eradicate PD-L1 expressing immunosuppressive cells in the TME. Further preclinical validation of the principle is encouraged.

Author Contributions: J.J.W.W. carried out the experiments, processed the experimental data, performed analysis, drafted the manuscript, and designed the figures. P.K.S conceived the original idea, provided funding and resources, supervised the project, and contributed to the final version of the manuscript.

Funding: The present work was financially supported by South-Eastern Norway Health Authority (funding number: 2017068 (P.K.S.) and 2016023 (J.J.W.W.)), The Norwegian Radium Hospital Research Foundation (funding number: FU0803 (P.K.S), and RadForsk (funding number: SE2002 (J.J.W.W.)).

Acknowledgments: We would thank PCI Biotech for providing us with the PCI photosensitizer fimaporfin/TPCS 2 and Advanced Targeted Systems (ATS) for providing the anti-PD-L1-saporin immunotoxin. We would also like to thank the Hospital Pharmacy at Oslo University Hospital, Rikshospitalet, for providing us with unused atezolizumab (Tecentriq) from the Cancer Clinic. 


\section{Short communication}

Conflicts of Interest: J.J.W.W. declares no conflict of interest. P.K.S. is a co-inventor on several patents and patent applications related to the PCI technology.

\section{References}

1. Sharma, P.; Siddiqui, B.A.; Anandhan, S.; Yadav, S.S.; Subudhi, S.K.; Gao, J.; Goswami, S.; Allison, J.P. The Next Decade of Immune Checkpoint Therapy. Cancer Discov 2021, 11, 838-857, doi:10.1158/2159-8290.CD-20-1680.

2. Chen, L.; Han, X. Anti-PD-1/PD-L1 therapy of human cancer: past, present, and future. J Clin Invest 2015, 125, 3384-3391, doi:10.1172/JCI80011.

3. Tang, F.; Zheng, P. Tumor cells versus host immune cells: whose PD-L1 contributes to PD-1/PD-L1 blockade mediated cancer immunotherapy? Cell Biosci 2018, 8, 34, doi:10.1186/s13578-018-0232-4.

4. Curiel, T.J.; Wei, S.; Dong, H.; Alvarez, X.; Cheng, P.; Mottram, P.; Krzysiek, R.; Knutson, K.L.; Daniel, B.; Zimmermann, M.C., et al. Blockade of B7-H1 improves myeloid dendritic cell-mediated antitumor immunity. Nat Med 2003, 9, 562-567, doi:10.1038/nm863.

5. Kleinovink, J.W.; Marijt, K.A.; Schoonderwoerd, M.J.A.; van Hall, T.; Ossendorp, F.; Fransen, M.F. PD-L1 expression on malignant cells is no prerequisite for checkpoint therapy. Oncoimmunology 2017, 6, e1294299, doi:10.1080/2162402X.2017.1294299.

6. Topalian, S.L.; Hodi, F.S.; Brahmer, J.R.; Gettinger, S.N.; Smith, D.C.; McDermott, D.F.; Powderly, J.D.; Carvajal, R.D.; Sosman, J.A.; Atkins, M.B., et al. Safety, activity, and immune correlates of anti-PD-1 antibody in cancer. N Engl J Med 2012, 366, 24432454, doi:10.1056/NEJMoa1200690.

7. Herbst, R.S.; Soria, J.C.; Kowanetz, M.; Fine, G.D.; Hamid, O.; Gordon, M.S.; Sosman, J.A.; McDermott, D.F.; Powderly, J.D.; Gettinger, S.N., et al. Predictive correlates of response to the anti-PD-L1 antibody MPDL3280A in cancer patients. Nature 2014, 515, 563-567, doi:10.1038/nature14011.

8. Powles, T.; Eder, J.P.; Fine, G.D.; Braiteh, F.S.; Loriot, Y.; Cruz, C.; Bellmunt, J.; Burris, H.A.; Petrylak, D.P.; Teng, S.L., et al. MPDL3280A (anti-PD-L1) treatment leads to clinical activity in metastatic bladder cancer. Nature 2014, 515, 558-562, doi:10.1038/nature13904.

9. Sharma, P.; Allison, J.P. The future of immune checkpoint therapy. Science 2015, 348, 56-61, doi:10.1126/science.aaa8172.

10. Brahmer, J.R.; Tykodi, S.S.; Chow, L.Q.; Hwu, W.J.; Topalian, S.L.; Hwu, P.; Drake, C.G.; Camacho, L.H.; Kauh, J.; Odunsi, K., et al. Safety and activity of anti-PD-L1 antibody in patients with advanced cancer. $N$ Engl J Med 2012, 366, 2455-2465, doi:10.1056/NEJMoa1200694.

11. Kalbasi, A.; Ribas, A. Tumour-intrinsic resistance to immune checkpoint blockade. Nat Rev Immunol 2020, 20, 25-39, doi:10.1038/s41577-019-0218-4.

12. Nowicki, T.S.; Hu-Lieskovan, S.; Ribas, A. Mechanisms of Resistance to PD-1 and PDL1 Blockade. Cancer J 2018, 24, 47-53, doi:10.1097/PPO.0000000000000303.

13. Burr, M.L.; Sparbier, C.E.; Chan, Y.C.; Williamson, J.C.; Woods, K.; Beavis, P.A.; Lam, E.Y.N.; Henderson, M.A.; Bell, C.C.; Stolzenburg, S., et al. CMTM6 maintains the expression of PD-L1 and regulates anti-tumour immunity. Nature 2017, 549, 101-105, doi:10.1038/nature23643. 


\section{Short communication}

14. Li, C.W.; Lim, S.O.; Chung, E.M.; Kim, Y.S.; Park, A.H.; Yao, J.; Cha, J.H.; Xia, W.; Chan, L.C.; Kim, T., et al. Eradication of Triple-Negative Breast Cancer Cells by Targeting Glycosylated PD-L1. Cancer Cell 2018, 33, 187-201 e110, doi:10.1016/j.ccell.2018.01.009.

15. Yao, H.; Lan, J.; Li, C.; Shi, H.; Brosseau, J.P.; Wang, H.; Lu, H.; Fang, C.; Zhang, Y.; Liang, L., et al. Inhibiting PD-L1 palmitoylation enhances T-cell immune responses against tumours. Nat Biomed Eng 2019, 3, 306-317, doi:10.1038/s41551-019-0375-6.

16. Phillip C.C. Liu, R.W., Liangxing Wu, Alla Volgina, Nina Zolotarjova, Luping Lin, Pramod Thekkat, Alex Margulis, Ronald Klabe, Wenqing Yao, Kaijiong Xiao, Jingwei Li, Xin He, Mark Rupar, Hong Chang, Paul Waeltz, Yanlong Li, Peggy Scherle, Reid Huber, Gregory Hollis. Novel small-molecule antagonists of the PD-1/PD-L1 axis that mediate cell surface PD-L1 dimerization and internalization [abstract]. In Proceedings of American Association for Cancer Research Annual Meeting 2019, Atlanta, GA. Philadelphia (PA).

17. Weng, A.; Thakur, M.; von Mallinckrodt, B.; Beceren-Braun, F.; Gilabert-Oriol, R.; Wiesner, B.; Eichhorst, J.; Bottger, S.; Melzig, M.F.; Fuchs, H. Saponins modulate the intracellular trafficking of protein toxins. J Control Release 2012, 164, 74-86, doi:10.1016/j.jconrel.2012.10.002.

18. Selbo, P.K.; Sandvig, K.; Kirveliene, V.; Berg, K. Release of gelonin from endosomes and lysosomes to cytosol by photochemical internalization. Biochim Biophys Acta 2000, 1475, 307-313, doi:10.1016/s0304-4165(00)00082-9.

19. Muller, L.; Tunger, A.; Plesca, I.; Wehner, R.; Temme, A.; Westphal, D.; Meier, F.; Bachmann, M.; Schmitz, M. Bidirectional Crosstalk Between Cancer Stem Cells and Immune Cell Subsets. Front Immunol 2020, 11, 140, doi:10.3389/fimmu.2020.00140.

20. Hsu, J.M.; Xia, W.; Hsu, Y.H.; Chan, L.C.; Yu, W.H.; Cha, J.H.; Chen, C.T.; Liao, H.W.; Kuo, C.W.; Khoo, K.H., et al. STT3-dependent PD-L1 accumulation on cancer stem cells promotes immune evasion. Nat Commun 2018, 9, 1908, doi:10.1038/s41467-01804313-6.

21. Terzuoli, E.; Bellan, C.; Aversa, S.; Ciccone, V.; Morbidelli, L.; Giachetti, A.; Donnini, S.; Ziche, M. ALDH3A1 Overexpression in Melanoma and Lung Tumors Drives Cancer Stem Cell Expansion, Impairing Immune Surveillance through Enhanced PDL1 Output. Cancers (Basel) 2019, 11, doi:10.3390/cancers11121963.

22. Zhang, C.; Wang, H.; Wang, X.; Zhao, C.; Wang, H. CD44, a marker of cancer stem cells, is positively correlated with PD-L1 expression and immune cells infiltration in lung adenocarcinoma. Cancer Cell Int 2020, 20, 583, doi:10.1186/s12935-020-01671-4.

23. Lee, Y.; Shin, J.H.; Longmire, M.; Wang, H.; Kohrt, H.E.; Chang, H.Y.; Sunwoo, J.B. CD44+ Cells in Head and Neck Squamous Cell Carcinoma Suppress T-Cell-Mediated Immunity by Selective Constitutive and Inducible Expression of PD-L1. Clin Cancer Res 2016, 22, 3571-3581, doi:10.1158/1078-0432.CCR-15-2665.

24. Prager, B.C.; Xie, Q.; Bao, S.; Rich, J.N. Cancer Stem Cells: The Architects of the Tumor Ecosystem. Cell Stem Cell 2019, 24, 41-53, doi:10.1016/j.stem.2018.12.009.

25. Kreso, A.; Dick, J.E. Evolution of the cancer stem cell model. Cell Stem Cell 2014, 14, 275-291, doi:10.1016/j.stem.2014.02.006.

26. Šošić, L.; Selbo, P.K.; Kotkowska, Z.K.; Kündig, T.M.; Høgset, A.; Johansen, P. Photochemical Internalization: Light Paves Way for New Cancer Chemotherapies and Vaccines. Cancers 2020, 12, 165. 


\section{Short communication}

27. Selbo, P.K.; Weyergang, A.; Hogset, A.; Norum, O.J.; Berstad, M.B.; Vikdal, M.; Berg, $\mathrm{K}$. Photochemical internalization provides time- and space-controlled endolysosomal escape of therapeutic molecules. J Control Release 2010, 148, 2-12, doi:10.1016/j.jconrel.2010.06.008.

28. Berg, K.; Nordstrand, S.; Selbo, P.K.; Tran, D.T.; Angell-Petersen, E.; Hogset, A. Disulfonated tetraphenyl chlorin (TPCS2a), a novel photosensitizer developed for clinical utilization of photochemical internalization. Photochem Photobiol Sci 2011, 10, 1637-1651, doi:10.1039/c1pp05128h.

29. Agostinis, P.; Berg, K.; Cengel, K.A.; Foster, T.H.; Girotti, A.W.; Gollnick, S.O.; Hahn, S.M.; Hamblin, M.R.; Juzeniene, A.; Kessel, D., et al. Photodynamic therapy of cancer: an update. CA Cancer J Clin 2011, 61, 250-281, doi:10.3322/caac.20114.

30. Sultan, A.A.; Jerjes, W.; Berg, K.; Hogset, A.; Mosse, C.A.; Hamoudi, R.; Hamdoon, Z.; Simeon, C.; Carnell, D.; Forster, M., et al. Disulfonated tetraphenyl chlorin (TPCS2a)induced photochemical internalisation of bleomycin in patients with solid malignancies: a phase 1, dose-escalation, first-in-man trial. Lancet Oncol 2016, 17, 1217-1229, doi:10.1016/S1470-2045(16)30224-8.

31. Dechene, A.; Kasper, S.; Olivecrona, H.; Schirra, J.; Trojan, J. Photochemical internalization and gemcitabine combined with first-line chemotherapy in perihilar cholangiocarcinoma: observations in three patients. Endosc Int Open 2020, 8, E1878E1883, doi:10.1055/a-1276-6366.

32. Otterhaug, T.; Janetzki, S.; Welters, M.J.P.; Hakerud, M.; Nedberg, A.G.; Edwards, V.T.; Boekestijn, S.; Loof, N.M.; Selbo, P.K.; Olivecrona, H., et al. Photochemical Internalization Enhanced Vaccination Is Safe, and Gives Promising Cellular Immune Responses to an HPV Peptide-Based Vaccine in a Phase I Clinical Study in Healthy Volunteers. Front Immunol 2020, 11, 576756, doi:10.3389/fimmu.2020.576756.

33. Latchman, Y.; Wood, C.R.; Chernova, T.; Chaudhary, D.; Borde, M.; Chernova, I.; Iwai, Y.; Long, A.J.; Brown, J.A.; Nunes, R., et al. PD-L2 is a second ligand for PD-1 and inhibits T cell activation. Nat Immunol 2001, 2, 261-268, doi:10.1038/85330.

34. Wong, J.J.W.; Berstad, M.B.; Fremstedal, A.S.V.; Berg, K.; Patzke, S.; Sorensen, V.; Peng, Q.; Selbo, P.K.; Weyergang, A. Photochemically-Induced Release of Lysosomal Sequestered Sunitinib: Obstacles for Therapeutic Efficacy. Cancers (Basel) 2020, 12, doi:10.3390/cancers12020417.

35. Olsen, C.E.; Cheung, L.H.; Weyergang, A.; Berg, K.; Vallera, D.A.; Rosenblum, M.G.; Selbo, P.K. Design, Characterization, and Evaluation of scFvCD133/rGelonin: A CD133-Targeting Recombinant Immunotoxin for Use in Combination with Photochemical Internalization. J Clin Med 2019, 9, doi:10.3390/jcm9010068.

36. Mittendorf, E.A.; Philips, A.V.; Meric-Bernstam, F.; Qiao, N.; Wu, Y.; Harrington, S.; Su, X.; Wang, Y.; Gonzalez-Angulo, A.M.; Akcakanat, A., et al. PD-L1 expression in triple-negative breast cancer. Cancer Immunol Res 2014, 2, 361-370, doi:10.1158/23266066.CIR-13-0127.

37. Eng, M.S.; Kaur, J.; Prasmickaite, L.; Engesaeter, B.O.; Weyergang, A.; Skarpen, E.; Berg, K.; Rosenblum, M.G.; Maelandsmo, G.M.; Hogset, A., et al. Enhanced targeting of triple-negative breast carcinoma and malignant melanoma by photochemical internalization of CSPG4-targeting immunotoxins. Photochem Photobiol Sci 2018, 17, 539-551, doi:10.1039/C7PP00358G.

38. Bostad, M.; Olsen, C.E.; Peng, Q.; Berg, K.; Hogset, A.; Selbo, P.K. Light-controlled endosomal escape of the novel CD133-targeting immunotoxin AC133-saporin by 
photochemical internalization - A minimally invasive cancer stem cell-targeting strategy. J Control Release 2015, 206, 37-48, doi:10.1016/j.jconrel.2015.03.008.

39. Berstad, M.B.; Cheung, L.H.; Berg, K.; Peng, Q.; Fremstedal, A.S.; Patzke, S.; Rosenblum, M.G.; Weyergang, A. Design of an EGFR-targeting toxin for photochemical delivery: in vitro and in vivo selectivity and efficacy. Oncogene 2015, 34, 5582-5592, doi:10.1038/onc.2015.15.

40. Passariello, M.; D'Alise, A.M.; Esposito, A.; Vetrei, C.; Froechlich, G.; Scarselli, E.; Nicosia, A.; De Lorenzo, C. Novel Human Anti-PD-L1 mAbs Inhibit ImmuneIndependent Tumor Cell Growth and PD-L1 Associated Intracellular Signalling. Sci Rep 2019, 9, 13125, doi:10.1038/s41598-019-49485-3.

41. Freeman, G.J.; Long, A.J.; Iwai, Y.; Bourque, K.; Chernova, T.; Nishimura, H.; Fitz, L.J.; Malenkovich, N.; Okazaki, T.; Byrne, M.C., et al. Engagement of the PD-1 immunoinhibitory receptor by a novel B7 family member leads to negative regulation of lymphocyte activation. J Exp Med 2000, 192, 1027-1034, doi:10.1084/jem.192.7.1027.

42. Juneja, V.R.; McGuire, K.A.; Manguso, R.T.; LaFleur, M.W.; Collins, N.; Haining, W.N.; Freeman, G.J.; Sharpe, A.H. PD-L1 on tumor cells is sufficient for immune evasion in immunogenic tumors and inhibits CD8 T cell cytotoxicity. J Exp Med 2017, 214, 895-904, doi:10.1084/jem.20160801.

43. Dougan, M.; Dranoff, G.; Dougan, S.K. Cancer Immunotherapy: Beyond Checkpoint Blockade. Annual Review of Cancer Biology 2019, 3, 55-75, doi:10.1146/annurevcancerbio-030518-055552.

44. Nguyen, L.T.; Ohashi, P.S. Clinical blockade of PD1 and LAG3--potential mechanisms of action. Nat Rev Immunol 2015, 15, 45-56, doi:10.1038/nri3790.

45. Tazzari, P.L.; Polito, L.; Bolognesi, A.; Pistillo, M.P.; Capanni, P.; Palmisano, G.L.; Lemoli, R.M.; Curti, A.; Biancone, L.; Camussi, G., et al. Immunotoxins containing recombinant anti-CTLA-4 single-chain fragment variable antibodies and saporin: in vitro results and in vivo effects in an acute rejection model. J Immunol 2001, 167, 42224229, doi:10.4049/jimmunol.167.8.4222.

46. Michot, J.M.; Bigenwald, C.; Champiat, S.; Collins, M.; Carbonnel, F.; Postel-Vinay, S.; Berdelou, A.; Varga, A.; Bahleda, R.; Hollebecque, A., et al. Immune-related adverse events with immune checkpoint blockade: a comprehensive review. Eur J Cancer 2016, 54, 139-148, doi:10.1016/j.ejca.2015.11.016.

47. Boutros, C.; Tarhini, A.; Routier, E.; Lambotte, O.; Ladurie, F.L.; Carbonnel, F.; Izzeddine, H.; Marabelle, A.; Champiat, S.; Berdelou, A., et al. Safety profiles of antiCTLA-4 and anti-PD-1 antibodies alone and in combination. Nat Rev Clin Oncol 2016, 13, 473-486, doi:10.1038/nrclinonc.2016.58.

48. Castano, A.P.; Demidova, T.N.; Hamblin, M.R. Mechanisms in photodynamic therapy: Part three-Photosensitizer pharmacokinetics, biodistribution, tumor localization and modes of tumor destruction. Photodiagnosis and Photodynamic Therapy 2005, 2, 91-106, doi:10.1016/S1572-1000(05)00060-8.

49. Emami, F.; Banstola, A.; Vatanara, A.; Lee, S.; Kim, J.O.; Jeong, J.H.; Yook, S. Doxorubicin and Anti-PD-L1 Antibody Conjugated Gold Nanoparticles for Colorectal Cancer Photochemotherapy. Mol Pharm 2019, 16, 1184-1199, doi:10.1021/acs.molpharmaceut.8b01157.

50. Sau, S.; Petrovici, A.; Alsaab, H.O.; Bhise, K.; Iyer, A.K. PDL-1 Antibody Drug Conjugate for Selective Chemo-Guided Immune Modulation of Cancer. Cancers (Basel) 2019, 11, doi:10.3390/cancers11020232. 


\section{Short communication}

51. Zhang, C.; Xiong, J.; Lan, Y.; Wu, J.; Wang, C.; Huang, Z.; Lin, J.; Xie, J. Novel cucurmosin-based immunotoxin targeting programmed cell death 1-ligand 1 with high potency against human tumor in vitro and in vivo. Cancer Sci 2020, 111, 31843194, doi:10.1111/cas.14549.

52. Polito, L.; Djemil, A.; Bortolotti, M. Plant Toxin-Based Immunotoxins for Cancer Therapy: A Short Overview. Biomedicines 2016, 4, doi:10.3390/biomedicines4020012.

53. Stratford, E.W.; Bostad, M.; Castro, R.; Skarpen, E.; Berg, K.; Hogset, A.; Myklebost, O.; Selbo, P.K. Photochemical internalization of CD133-targeting immunotoxins efficiently depletes sarcoma cells with stem-like properties and reduces tumorigenicity. Biochim Biophys Acta 2013, 1830, 4235-4243, doi:10.1016/j.bbagen.2013.04.033.

54. Selbo, P.K.; Rosenblum, M.G.; Cheung, L.H.; Zhang, W.; Berg, K. Multi-modality therapeutics with potent anti-tumor effects: photochemical internalization enhances delivery of the fusion toxin scFvMEL/rGel. PLoS One 2009, 4, e6691, doi:10.1371/journal.pone.0006691.

55. Weyergang, A.; Fremstedal, A.S.; Skarpen, E.; Peng, Q.; Mohamedali, K.A.; Eng, M.S.; Cheung, L.H.; Rosenblum, M.G.; Waltenberger, J.; Berg, K. Light-enhanced VEGF121/rGel: A tumor targeted modality with vascular and immune-mediated efficacy. J Control Release 2018, 288, 161-172, doi:10.1016/j.jconrel.2018.09.005.

56. Norum, O.J.; Fremstedal, A.S.V.; Weyergang, A.; Golab, J.; Berg, K. Photochemical delivery of bleomycin induces T-cell activation of importance for curative effect and systemic anti-tumor immunity. J Control Release 2017, 268, 120-127,

doi:10.1016/j.jconrel.2017.10.015. 


\section{Figure legends}

Figure 1. PD-L1 surface expression in the human cancer cell line panel

(A) Representative flow cytometry histograms of PD-L1 surface expression on MDA-MB-231, MDA-MB-453, MDA-MB-468, PC-3, DU145, and PANC-1 cells. Red line indicates secondary antibody alone and blue line indicates primary and secondary antibody (PD-L1 expression). (B) Quantification of the PD-L1 expression in the cell panel. The relative PD-L1 expression is the median fluorescence intensity of anti-PD-L1-stained sample relative to secondary antibody alone. Data presented as mean \pm S.E.M. of at least two independent experiments.

Figure 2. Specific binding, uptake and PCI-induced cytosolic release of PD-L1 antibody

(A) Fluorescence microscopy of cell surface binding of anti-PD-L1 antibody (PD-L1-targeting primary antibody bound to secondary antibody labelled with AlexaFluor488, Anti-PD-L1AF488, green signal) in live MDA-MB-231 cells. The cells were incubated on ice to prevent endocytosis. (B) Cellular uptake of anti-PD-L1-AF488 (green) in live MDA-MB-231 cells after overnight incubation (18 hours) at $37{ }^{\circ} \mathrm{C}$ and co-localization with LysoTracker Red (red) in live MDA-MB-231 cells. Co-localization of antibody and LysoTracker is indicated in merge (yellow/orange), with corresponding DIC image. (C) Upper four panels (No light = before PCI), Anti-PD-L1-AF488 (green) and TPCS2a (red) after 18 hours co-incubation and 4 hours chase, with corresponding merge and DIC images. Lower four panels (Light = PCI), fluorescence images of PCI-induced cytosolic release of internalized PD-L1 and TPCS $2 \mathrm{a}$ in live MDA-MB-231 cells. Co-localization (merge) of TPCS 2 and anti-PD-L1-AF488 indicated in yellow/orange, with corresponding DIC image. Nucleus (DNA) was stained with Hoechst 33342 (blue). Scale bar $=20 \mu \mathrm{m}$. Light exposure (LumiSource): 100 seconds $\approx 0.7 \mathrm{~J} / \mathrm{cm}^{2}$. All images are representative images of three independent experiments.

Figure 3. Specific and PCI-enhanced cytotoxicity of anti-PD-L1-saporin in PD-L1 $1^{\text {high }}$ MDAMB-231 cells

(A) Light dose-dependent cytotoxic responses to PCI. Relative cell viability of MDA-MB-231 after PCI of immunotoxin (anti-PD-L1-saporin) or the non-targeting toxin (saporin) compared to PDT $\left(0.2 \mu \mathrm{g} / \mathrm{mL}\right.$ TPCS $_{2 \mathrm{a}}+$ light $)$. Cells were incubated with $10 \mathrm{pM}$ of the immunotoxin or toxin for 4 (left panel) or 18 hours (right panel) prior to light exposure (1 $\min =0.42 \mathrm{~J} / \mathrm{cm}^{2}$ ). (B) Relative cell viability of PD-L1-negative MDA-MB-453 after PCI of 100 pM anti-PD-L1-saporin or saporin compared to PDT $\left(0.4 \mu \mathrm{g} / \mathrm{mL}\right.$ TPCS $_{2 a}+$ light). Representative experiments out of at least two independent experiments. Data presented as mean of triplicates \pm S.D. (C) Efficacy of immunotoxin and toxin in the absence of PCI. Cytotoxicity of anti-PD-L1-saporin or saporin in MDA-MB-231 cell incubated for 18 hours. The reference line indicates $50 \%$ relative cell viability. Data presented as mean of at least three independent experiments \pm S.E.M. (D) Concentration-dependent PCI of immunotoxin or toxin in MDA-MB-231 cells using a fixed light dose corresponding to 110 seconds $\approx 0.77$ 


\section{Short communication}

$\mathrm{J} / \mathrm{cm}^{2}$. (E) Atezolizumab (Tecentriq) does not enhance the PDT-efficacy. No enhanced efficacy was achieved by combining PD-L1 blockade with PDT $\left(0.2 \mu \mathrm{g} / \mathrm{mL}\right.$ TPCS $2_{2}+$ light). Left panel, Increasing atezolizumab concentrations in combination with fimaporfin/TPCS $\mathrm{S}_{2 \mathrm{a}}$ exposed to 110 seconds light. Right panel, increasing light doses of $1 \mu \mathrm{M}$ atezolizumab combined with fimaporfin, both co-incubated for 18 hours. For all experiments, cytotoxicity was evaluated 48 hours post-light exposure using the MTT assay. Representative experiments out of at least three independent experiments. Data presented as mean of triplicates \pm S.D.

\section{Figure 4. IFN- $\gamma$ induce surface expression of PD-L1 in murine colon cancer cells}

(A) Representative flow cytometry histogram of surface PD-L1 expression in the murine colon cancer cell lines CT26.WT and MC-38. Red line indicates secondary antibody alone, blue line indicates primary and secondary antibody (PD-L1 expression), and orange line indicates IFN- $\gamma$ treated cells (100 U/ml, 24 hours). (B) Quantification of the PD-L1 expression in CT26.WT and MC-38. The relative PD-L1 expression is the median fluorescence intensity of stained sample relative to secondary antibody alone. Data presented as mean \pm S.E.M. of at least two independent experiments. Statistical significance calculated using student twotailed t-test $\left.{ }^{* * *} \mathrm{p} \leq 0.001,{ }^{*} \mathrm{p} \leq 0.05\right)$. 

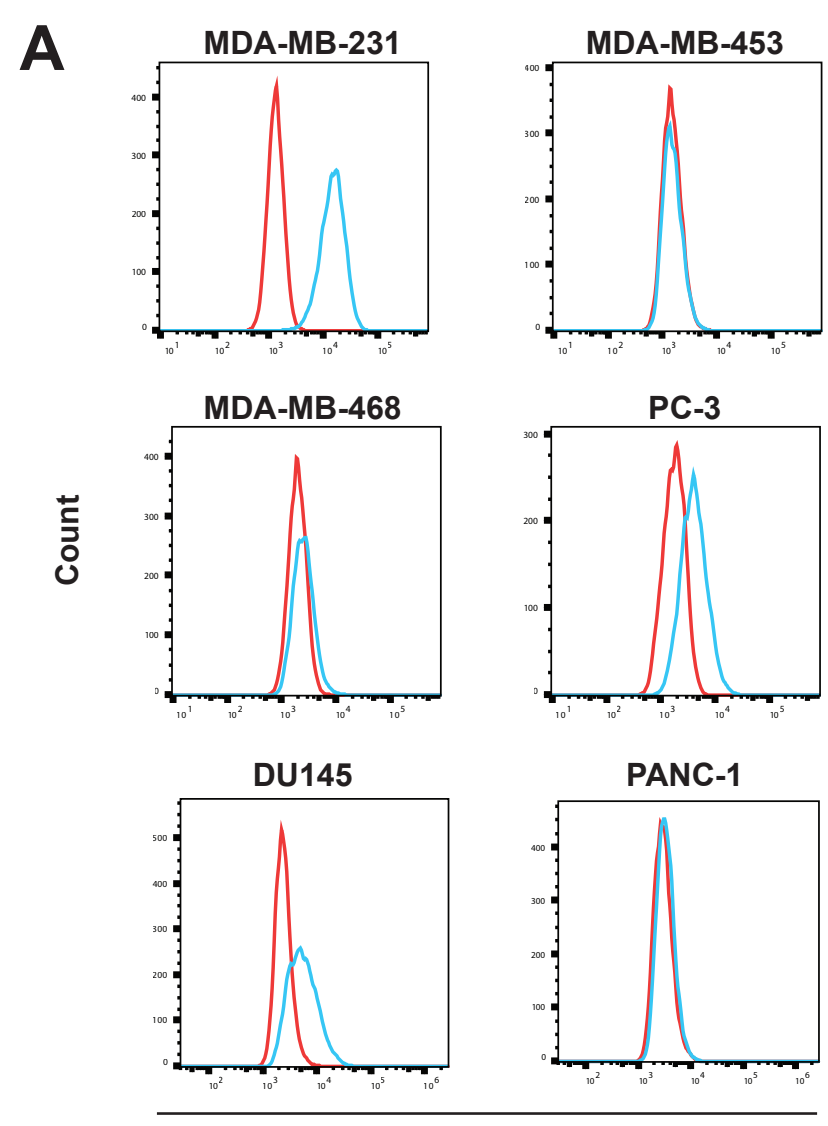

anti-PD-L1-AF488

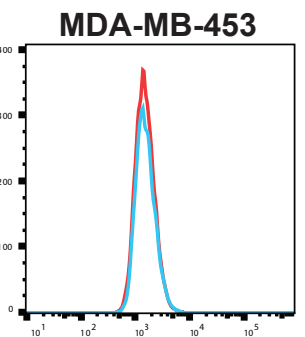

PC-3

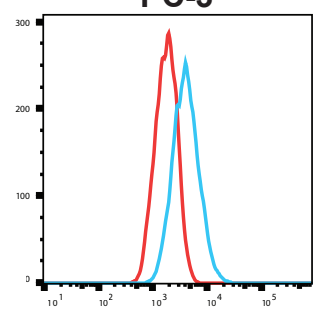

PANC-1

Secondary antibody

PD-L1 expression 

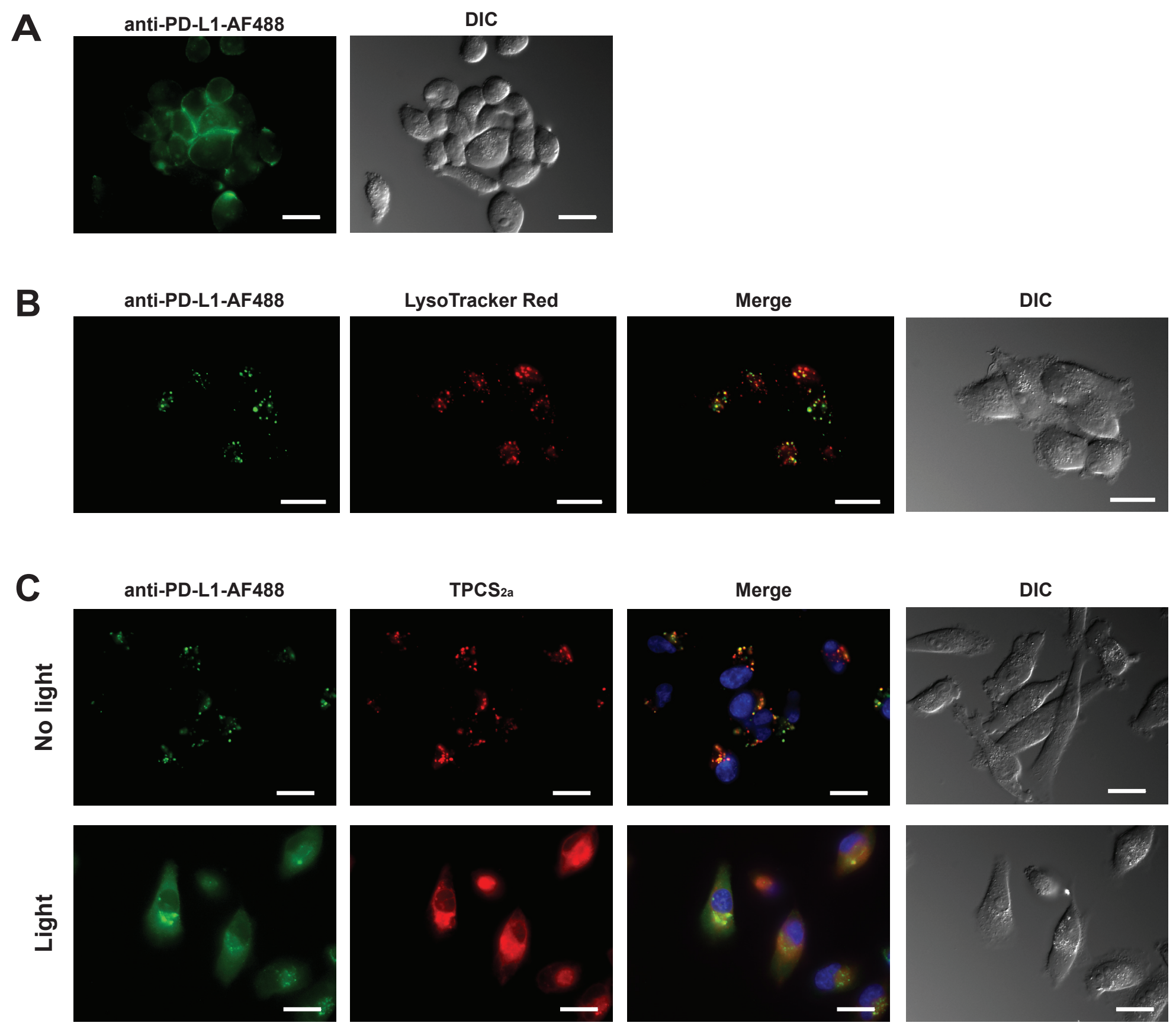
A

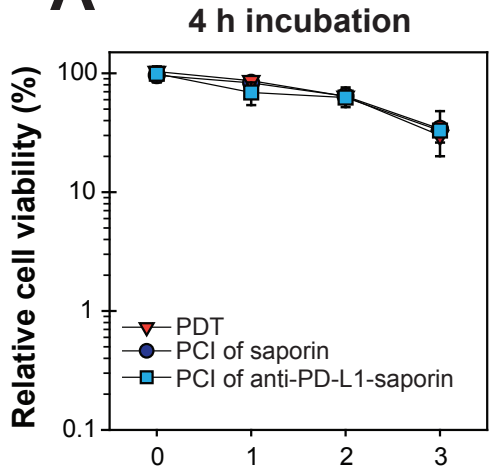

Light exposure (minutes)
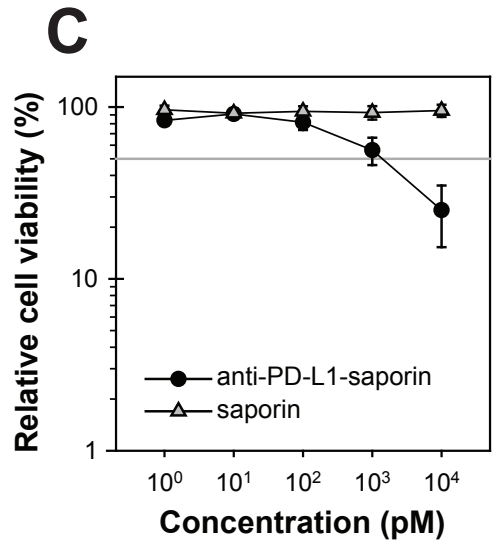

E

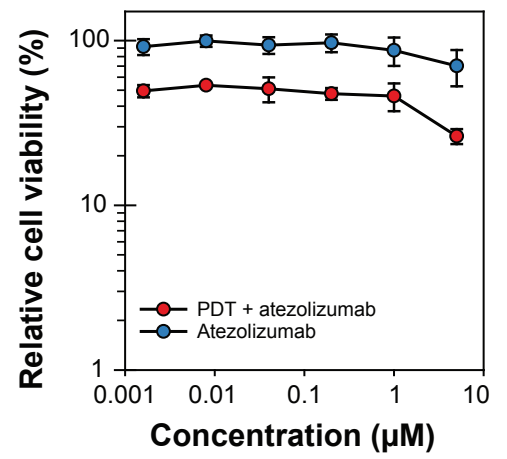

B

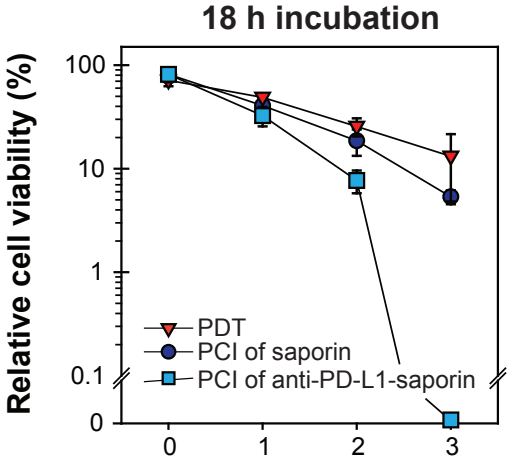

Light exposure (minutes)

D
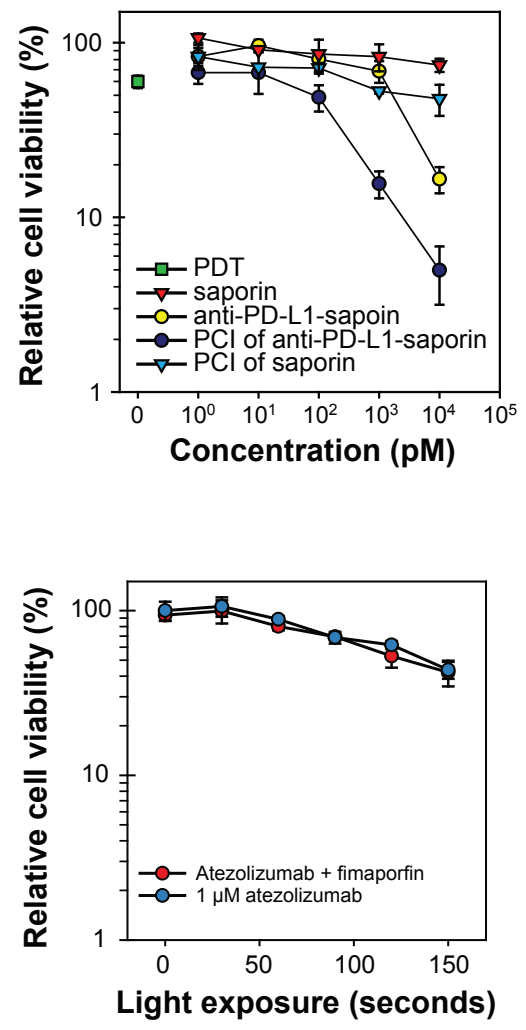

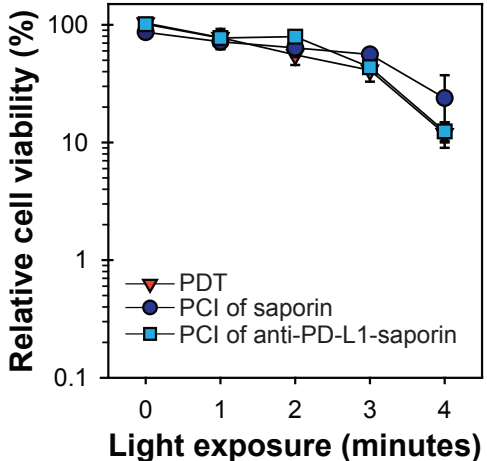

Light exposure (minutes) 
A

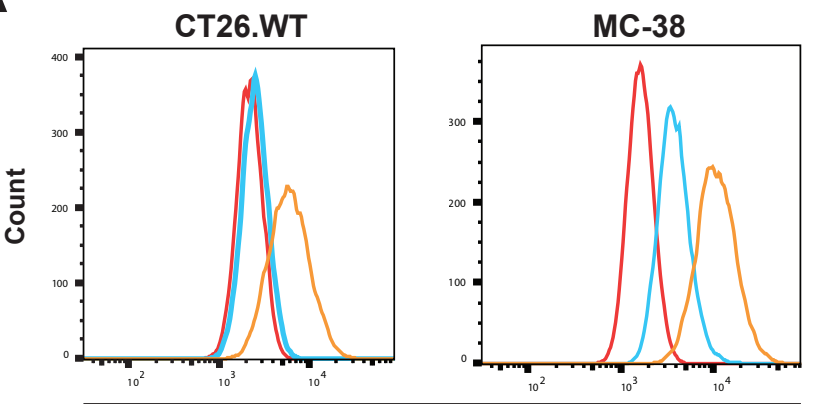

anti-PD-L1-AF488

Secondary antibody

Basal PD-L1 expression

IFN- $y$-induced PD-L1 expression
B

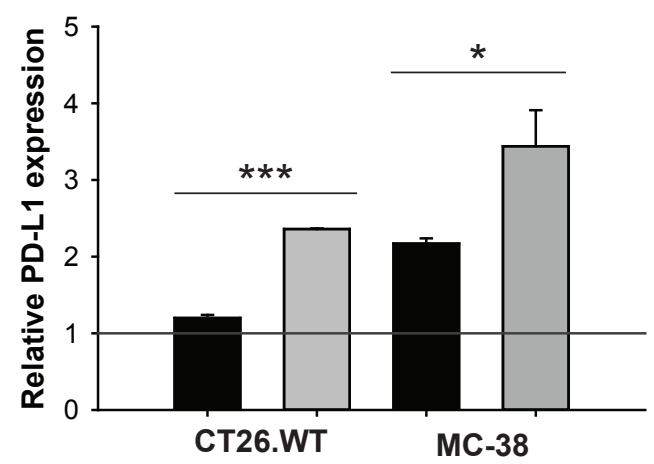

Basal PD-L1 expression

$\square$ IFN-y-induced PD-L1 expression 